\title{
The treatment of integrated traditional Chinese and Western medicine to cure severe immune thrombocytopenia in a patient in a minimally conscious state: a case report
}

\author{
Yu Wang ${ }^{1 \#}$, Liping Zhang ${ }^{2 \#}$, Julong Tian ${ }^{1}$, Ma Lan ${ }^{1,2}$, Yunshi Liu ${ }^{1}$, Tianling Yan ${ }^{1}$, Qinghua Qiu ${ }^{1}$ \\ ${ }^{1}$ Panzhihua University, Affiliated Hospital of Panzhihua University, Panzhihua, China; ${ }^{2}$ Panzhihua University, Health and Wellness College, \\ Panzhihua, China \\ \#These authors contributed equally to this work and are co-first authors. \\ Correspondence to: Julong Tian. Affiliated Hospital of Panzhihua University, Panzhihua, China. Email: 551333202@qq.com.
}

\begin{abstract}
Immune thrombocytopenia is a common complication in patients in a minimally conscious state (MCS). MCS patients are prone to pulmonary infection for the reasons of long-term bed rest and tracheotomy etc., which leads to frequent immune thrombocytopenia. At present, there is no specific treatment for immune thrombocytopenia. Moreover, the cost of routine treatment is high, and clinicians need to consider different drug combinations, side effects, and the risk of drug dependence when selecting treatments. Here, we report a case of a patient in a MCS who developed immune thrombocytopenia after tracheotomy and long-term bed laying in October 2015. The patient's platelet count declined continuously, and by December 2015, she was in a critical condition, with a platelet count of less than $20 \times 10^{9} / \mathrm{L}$. The patient firstly received routine treatment, however, this could only temporarily prevent the drop in platelets. Following a series of explorations, the patient was treated with a combination of traditional Chinese and Western medicine, which included treatment and preventive measures. For treatment, the patient was given roxithromycin dispersible administration tablets and a self-made preparation of peanut red skin, which could quickly cure the immune thrombocytopenia. Preventive measures included the addition of ursodeoxycholic acid capsules, silybin capsules, and a traditional Chinese medicine preparation. As shown by laboratory examination results, the patient's platelet count has stayed around a normal level since March 2016, and she now has normal liver and kidney function. This outcome evidence that combined traditional Chinese and Western medicine could effectively cure immune thrombocytopenia and prevent its recurrence. Moreover, the cost of the treatment was lower and there were fewer side effects than routine treatment, and at the same time, the method of treatment was simple and convenient. Our practical experience may provide a valuable clue for the treatment of immune thrombocytopenia.
\end{abstract}

Keywords: Minimally conscious state (MCS); immune thrombocytopenia; peanut red skin

Submitted Dec 14, 2020. Accepted for publication Mar 11, 2021.

doi: 10.21037/apm-21-307

View this article at: http://dx.doi.org/10.21037/apm-21-307

\section{Introduction}

Minimally conscious state (MCS), which is also referred to as the tiny state of consciousness, is a chronic condition characterized by a severe disturbance of consciousness. Patients in an MCS lose the basic cognitive ability about their own and external environment, keeping only some instinct nerve reflex and material energy metabolism (1). Patients in an MCS can easily develop pulmonary infection due to loss or weakening of body function caused by longterm bed laying and tracheotomy (2). The latest research revealed platelet apoptosis caused by pathogenic infection to be the key factor of thrombocytopenia (3). For instance, studies have evidenced that mycoplasma infection can cause immune thrombocytopenia, which is related to an increase in condensate caused by the infection. Condensate is a type of polyclonal IgM antibody, which can be condensed on the 
surface of platelets to produce corresponding antibodies, resulting in a cross reaction between the antibodies and platelet membrane that damages platelets $(4,5)$.

Immune thrombocytopenia refers to thrombocytopenia caused by viral, bacterial, or other infections. Viruses can invade megakaryocytes and reduce the production of platelets. They can also attach to platelets, increasing platelet destruction. Bacterial toxins inhibit platelet production, or increase platelet destruction and increase platelet consumption due to toxins affecting vascular wall function (6). Yang observed 34 patients hospitalized with pulmonary infection and reported that the proportion of patients who progressed to moderate and severe thrombocytopenia was about $47 \%$ and $30 \%$ respectively (7). When the platelet count is less than $100 \times 10^{9} / \mathrm{L}$, it is defined as Thrombocytopenia, mild, moderate, severe and extremely severe thrombocytopenia are judged according to the platelet count of $50 \times 10^{9} / \mathrm{L}$ to $100 \times 10^{9} / \mathrm{L}, 30 \times 10^{9} / \mathrm{L}$ to $50 \times 10^{9} / \mathrm{L}$, $10 \times 10^{9} / \mathrm{L}$ to $30 \times 10^{9 /} \mathrm{L}$, less than $10 \times 10^{9} / \mathrm{L}$ respectively (8). Mild to moderate thrombocytopenia has no obvious symptoms or signs; however, severe and extremely severe thrombocytopenia carries a high risk of visceral hemorrhage and intracranial hemorrhage (9). Currently, the routine treatment for immune thrombocytopenia mainly includes fighting infection, strengthening immunity, and promoting platelet production. At present, there is no specific treatment for the disease. In the meantime, when selecting treatments, clinicians must consider different drug combinations, complications, side effects, and the risk of drug dependence (10-12). Therefore, the treatment of immune thrombocytopenia is an issue of concern around the world, especially in patients in an MCS.

Here, we report the case of an MSC patient in our hospital who developed severe thrombocytopenia in December 2015. The treating physician adopted a combination treatment of traditional Chinese and Western medicine to cure and prevent the recurrence of immune thrombocytopenia, which achieved great results. Peanut red skin played an important role in the successful treatment of the patient. Existing studies have found that peanut red coating and its extract contain a variety of functional components, such as antibacterial, antioxidant, and other active components, which have been recorded in the National Compilation of Chinese Herbal Medicine (13). Peanut red skin also has the effect of preventing thrombocytopenia. This study is reported according to the principle of People-oriented, equal emphasis on traditional Chinese and Western medicine. We present the following article in accordance with the CARE reporting checklist (available at http://dx.doi.org/10.21037/ apm-21-307).

\section{Case presentation}

A 56-year-old (1955-01) female (Civil Service) was sent to our hospital in April 2011 with sudden severe pain in the head without an obvious cause. The patient was diagnosed with ventricular hemorrhage, and given craniocerebral surgery immediate, including outdoor of bilateral lateral ventricular drainage, posterior fossa craniotomy, cerebrovascular malformation resection, removal of the fourth ventricle hematoma, continuous drainage of the lumbar cistern, and other surgical procedures. Through a series of emergent effective treatments, the patient's life was saved but in a state of minimal consciousness. In October 2015, the patient developed thrombocytopenia of unknown cause. From October to November 2015, the patient's platelet count decreased to $(50-100) \times 10^{9} / \mathrm{L}$. On December 14th, 2015, the patient's platelet count dropped to $40 \times 10^{9} / \mathrm{L}$. On December 15 th, 2015 , the nurse found some blood filament in the sputum of the patient. The result of laboratory examination revealed the platelet count was $17 \times 10^{9} / \mathrm{L}$, indicating severe thrombocytopenia. The patient had no history of drugs allergies or family history. All procedures performed in studies involving human participants were in accordance with the ethical standards of the institutional and/or national research committee(s) and with the Helsinki Declaration (as revised in 2013). Written informed consent was obtained from the patient.

\section{Diagnosis}

Many factors contribute to thrombocytopenia, such as HenochSchonlein purpura and Thrombotic thrombocytopenic purpura. Pathogenic examination of the patient was performed on December 15 th, 2015. The results of Respiratory Tract Profile (IgM) examinations showed positivity for the pneumonia mycoplasma IgM antibody, and enzyme-linked immunosorbent assay (ELISA) showed $\mathrm{B}_{19}$ virus IgM antibody positivity. Therefore, pneumonia mycoplasma and $B_{19}$ virus were determined to be the causes of thrombocytopenia in this patient. Immune Thrombocytopenia associated with pulmonary infection was considered.

\section{Treatment and outcomes}

\section{Routine treatment}

\section{Strengthen immunity and anti-infection therapy}

The treating doctor administered the patient human immunoglobulin (HIG), recombinant human granulocyte 


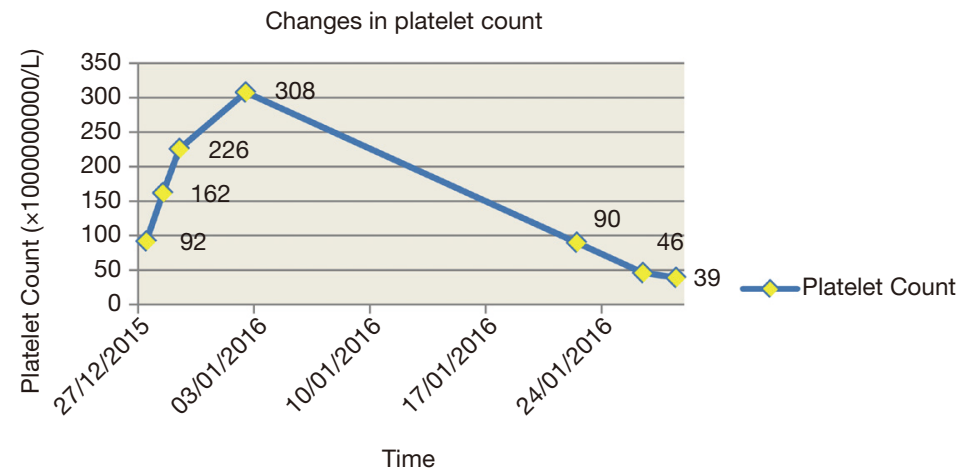

Figure 1 The effect of routine treatment on the platelet count of the patient.

coloery-stimulating factor injection (RHGSFI), and roxithromycin dispersible administration tablets (RDATs) to enhance her immunity and control the infection. Roxithromycin has an excellent killing effect on mycoplasma. No specific treatment exists for $\mathrm{B}_{19}$ virus infection, and the treatment mainly includes antiviral therapy and enhance immunity. Our patient received HIG at a dose of $10 \mathrm{~g}$ $(2.5 \mathrm{~g} /$ bottle $\times 4$ bottles) via intravenous drip on December 12th and 13th, 2015 respectively. On December 17th, 2015, the patient's platelet count declined to $13 \times 10^{9} / \mathrm{L}$, and she was consequently administered emergency dose of HIG ( $45 \mathrm{~g}, 2.5 \mathrm{~g} / \mathrm{bottle} \times 18$ bottles). The next day, HIG was given once again, at a dose of $22.5 \mathrm{~g}(2.5 \mathrm{~g} /$ bottle $\times 9$ bottles $)$. The patient received RHGSFI at a dose of $75 \mu \mathrm{g}$ via hypodermic injection on December 13th, 15th, 26th, and 31th, 2015, and on January 17th and 25th, 2016. She was also given RDATs via fistula tube injection between December 12th, 2015 and January 25th, 2016, twice a day with 1 tablet each time.

\section{Promotion of platelet production and supportive treatment}

In order to promote platelet production, the patient was given recombinant human thrombogenic injection (RHTI). From December 15th to 28th, 2015, she received RHTI by hypodermic injection (once a day, 7,500 U/day). On December 17th, 2015, the patient's platelet count decreased to $13 \times 10^{9} / \mathrm{L}$, she was given a therapeutic dose of O-type blood platelets via intravenous drip on December 17 th, 19th, and 21st, 2015 respectively.

\section{Therapeutic effect}

The routine treatment could only temporarily maintain the platelet count at a normal level. It took about two weeks for the patient's platelet count to return to a normal level, and it remained at a normal level for less than 1 month (approximately 27 days). The patient was treated with the routine treatment from December 12th, 2015, and on December 27 th, the platelet count was approaching a normal level $\left(91 \times 10^{9} / \mathrm{L}\right)$. It peaked at $308 \times 10^{9} / \mathrm{L}$ on January 2nd, 2016, and then began to decline continually, dropping below $100 \times 10^{9} / \mathrm{L}$ on January $22 \mathrm{nd}$, and further declining to $39 \times 10^{9} / \mathrm{L}$ on January 28 th (Figure 1 ).

\section{Combined treatment with traditional Chinese and Western medicine}

\section{Treatment measures}

The treatment combined traditional Chinese and Western medicine, including the following measures: (I) the patient continued to take RDATs to combat infection (twice a day with 1 tablet each time); (II) received RHGSFI at a dose of $75 \mu \mathrm{g}$ via hypodermic injection on February 6th, 2016; (III) given a self-made preparation of peanut red skin starting on January 28th, 2016 (twice a day at a dose of $150 \mathrm{~mL}$ each time). The preparation consisted of $100 \mathrm{~g}$ of red peanut skin, to which $1,500-2,000 \mathrm{~mL}$ water was added to soak for 30 minutes, followed by simmering for 40 minutes to extract $450 \mathrm{~mL}$ preparation.

\section{Therapeutic effect}

The treatment of integrated traditional Chinese and Western medicine effectively cured immune thrombocytopenia in the patient. It took 3 days to take effect and 15 days to reach the normal level. After the patient began taking the peanut red skin preparation on January 28th, 2016, her platelet count increased from $39 \times 10^{9} / \mathrm{L}$ to $56 \times 10^{9} / \mathrm{L}$ on February 1st, 2016. The platelet count continued to rise to a normal level, increasing to $107 \times 10^{9} / \mathrm{L}$ on February 12 th and then 


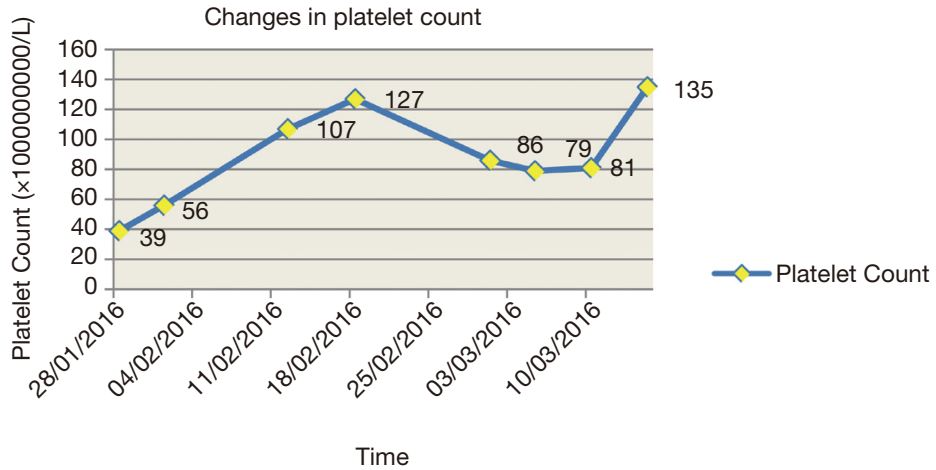

Figure 2 The effect of combined treatment on the platelet count of the patient.

remaining at around $100 \times 10^{9} / \mathrm{L}$ thereafter, with some small fluctuations. The patient's laboratory examination results showed that the platelet count was $127 \times 10^{9} / \mathrm{L}, 86 \times 10^{9} / \mathrm{L}$, $79 \times 10^{9} / \mathrm{L}, 81 \times 10^{9} / \mathrm{L}$, and $135 \times 10^{9} / \mathrm{L}$ on February 18 th, and March 1st, 5th, 10th, and 15th, 2016, respectively (Figure 2).

\section{Preventive measures}

Patients with immune thrombocytopenia are prone to relapse, which complicates treatment and makes longterm therapy necessary (14). Moreover, the current case involved a patient in an MCS. In order to prevent the recurrence of thrombocytopenia caused by pulmonary infection, preventive measures also adopted to combine traditional Chinese and Western medicine. The preventive measures included the addition of ursodeoxycholic acid capsules, silybin capsules, and a traditional Chinese medicine preparation on the basis of the RDATs, a peanut red skin preparation and the traditional Chinese medicine preparation. The patient received RDATs (1 tablet, twice a day) to prevent the recurrence of pulmonary mycoplasma infection, as well as ursodeoxycholic acid capsules and silybin capsules (1 pill/day for both) to reduce liver damage from roxithromycin. At the same time, a peanut red skin preparation was given to stimulate platelet production. The traditional Chinese medicine preparation given to the patient had the affect of fighting infection, enhancing immunity, reinforcing energy, enriching the blood, and regulating the intestines and stomach (Table 1). Our patient took $200 \mathrm{~mL}$ of the preparation 3 times a day. A dose of traditional Chinese medicine could be used for 3 to 5 days. Since using the preparation, the platelet count of the patient has stayed around a normal level, and recent laboratory examination results showed that her liver and kidney function has returned to normal (from March 2016 to now).

\section{Discussion}

Immune thrombocytopenia is a common complication in patients in an MCS. The normal structure of the respiratory tract is destroyed for tracheotomy, the respiratory tract exposes to air for a long time, the discharge of airway secretions is blocked and active substances on the lung surface are destroyed because of without the ability to cough autonomously, thus increasing the risk of respiratory tract infection (14). Mycoplasma pneumonia is particularly tricky to eradicate, and patients with low immunity are prone to repeated infection, while mycoplasma can directly inhibit megakaryocytes to reduce platelet production (15). No specific treatment measures currently exist for immune thrombocytopenia. Therefore, the treatment and prevention of immune thrombocytopenia in patients in an MCS is a serious and challenging clinical issue.

The research results showed that the combined treatment with traditional Chinese and Western medicine had four advantages over routine treatment. The routine treatment adopted HIG, RHGSFI, and RDATs to improve the body's immunity and achieve an anti-infective effect, as well as RHTI and type-O blood platelet treatment to improve the platelet count. The treatment integrated Chinese traditional and Western medicine, mainly in the form of roxithromycin and peanut red coat preparation. The combined treatment had a greater therapeutic effect than routine treatment. The patient initiated the prior treatment on January 28th, 2016; it took only 3 days to take effect, and the platelet count rose and then has stayed at around $100 \times 10^{9} / \mathrm{L}$. However, the routine treatment could only maintain the platelet count at a normal level for less than 1 month. The combined treatment also cost less than the routine treatment, with the latter costing around several 
Table 1 Traditional Chinese medicine prescription pad

\begin{tabular}{|c|c|c|}
\hline Number & Chinese herbal medicine & Dose (g) \\
\hline 1 & Garden Burnet Root & 15 \\
\hline 2 & Herba Ecliptae & 15 \\
\hline 3 & Glossy Privet Fruit & 15 \\
\hline 4 & Arisaema Cum Bile & 10 \\
\hline 5 & Forsythia Suspensa & 15 \\
\hline 6 & Licorice Root & 10 \\
\hline 7 & Chinese Thorowax Root & 20 \\
\hline 8 & Cogongrass Rhizome & 30 \\
\hline 9 & Bitter Apricot Kernel & 15 \\
\hline 10 & Medicinal Fermented Mass & 10 \\
\hline 11 & Honeysuckle Flower & 15 \\
\hline 12 & Cyclamen & 15 \\
\hline 13 & Wrinkled Giant Hyssop Herb & 15 \\
\hline 14 & Glehniae Radix & 30 \\
\hline 15 & Rock White Mulberry & 30 \\
\hline 16 & Heartleaf Houttuyniae Herb & 10 \\
\hline 17 & Herba Oldenlandia & 30 \\
\hline 18 & Common Aucklandiae Root & 15 \\
\hline 19 & Thunberg Fritillary Bulb & 15 \\
\hline 20 & Amomi Rotundus & 15 \\
\hline 21 & Bamboo Shavings & 30 \\
\hline 22 & Reed Rhizome & 15 \\
\hline 23 & Chicken Gizzard Membrane & 10 \\
\hline 24 & Barbary Wolfberry Fruit & 25 \\
\hline 25 & Immature Orange Fruit & 20 \\
\hline 26 & Baical Skullcap Root & 15 \\
\hline 27 & Dwarf Lilyturf Tuber & 15 \\
\hline 28 & Officinal Magnolia Bark & 10 \\
\hline 29 & Polygonatum Sibiricum & 15 \\
\hline 30 & Lophatherum Gracile & 15 \\
\hline 31 & Indigowoad Root & 15 \\
\hline 32 & Cortex Phellodendri & 15 \\
\hline 33 & Radix Rehmanniae Preparata & 15 \\
\hline
\end{tabular}

hundred RMB per day, compared to 10 RMB per day for the former. Furthermore, there were fewer side effects compares with the routine treatment. Peanut red skin is both medicine and food, meaning it has almost no side effects. Finally, the combined treatment method was simpler, because only RDATs were combined with the peanut red skin preparation, and both are orally administered. Therefore, these treatment measures were simple and convenient.

Peanut red skin preparation plays an important role in integrated traditional Chinese and Western medicine. The medicinal value of peanut red skin has been gradually recognized, and it is currently applied in clinic. Chen used peanut red skin to treat 38 patients with thrombocytopenic purpura; the treatment cured 30 patients, achieved an improvement in 5 patients, and failed to cure 3 patients, with the total effective rate reaching $92.1 \%$ after 1 course of treatment (16). The related studies confirmed that the active ingredients extracted from the peanut red skin contained procyanidins, forulic acid, resveratrol, flavone, catechins, and coumarin elements, which have the effects of antioxidant, antibacterial, antiviral, anticancer, and antimutagenesis, also have the functions of regulating the immune system, strengthening the resistance of capillaries, promoting bone marrow hematopoiesis and reducing platelet loss $(17,18)$. Gao's study demonstrated that peanut red skin could play a positive role in improving thrombocytopenia caused by chemotherapy via inhibiting platelet apoptosis through the down-regulation of Bax and Bak protein expression (19).

According to the basic theory of traditional Chinese medicine, blood following the pulse normal is inseparable from the solid role of Qi. Qi can govern blood, which is mainly reflected in the physiological function of spleen Qi in controlling blood. Sufficient spleen Qi can guarantee the normal operation of blood and the function of nourishing. However, if spleen Qi becomes weakened and loses the governing, it can also lead to a variety of bleeding lesions, such as subcutaneous bleeding, hematochezia, blood urination, metrorrhagia, and metrostaxis, as well as other bleeding symptoms; this is known as "syndrome of qi failing to control blood" or "spleen failing to manage blood". Therefore, when treating these bleeding lesions, we must use the method of invigorating the spleen and qi to replenish qi in order to govern blood. Peanut red skin, which is called 
red-skin in traditional Chinese medicine, is recorded in the National Compilation of Chinese Herbal Medicine. it is "mainly used for hemostasis, dispersing blood stasis, and detumescence" in the treatment of various bleeding symptoms. Peanut red skin is rich in polyphenols and is known as "the seventh nutrient (13,17-21).

Roxithromycin represents a new generation of macrolides antibiotics, which mainly acts on gram-positive bacteria, anaerobic bacteria, chlamydia, and mycoplasma. Its antibacterial effect in vitro is similar to that of erythromycin, and its antibacterial effect in vivo is 1-4 times stronger than that of erythromycin (22). It has been used to treat bronchitis, pneumonia, tonsillitis, five sense organ infections, urinary system infections, and skin and soft tissue infections caused by sensitive bacteria. Roxithromycin has a great effect in killing mycoplasma. In our patient, laboratory results showed that the pathogenic bacteria responsible for the pulmonary infection were mycoplasma and $\mathrm{B}_{19}$. The patient subsequently received RDATs instead of roxithromycin capsules. RDATs have the characteristics of convenient administration, rapid disintegration, good absorption, and a rapid effect compared with roxithromycin capsules. They can be completely dispersed within 3 to 5 minutes after exposure water, which makes them especially useful in the treatment of patients who have difficulty swallowing. However, long-term administration of roxithromycin can result in damage to liver function and a rise in transaminase (22). Ursodeoxycholic acid capsules and silybin capsules were administered to protect the patient's liver and improve the liver detoxification function during the administration of roxithromycin. Ursodeoxycholic acid capsule is mainly used for the treatment of cholestatic liver disease and has a certain protective effect on the liver (23). In liver cells, silibin capsules can stabilize the membrane, protect the enzyme system, and remove active oxygen free radicals, which can significantly improve liver detoxification function (24). The patient in the current study has been receiving RADTs combined with ursodeoxycholic acid capsules and silybin capsules for more than 3 years, and recent laboratory examination results have shown her liver function to be normal.

Patients with immune thrombocytopenia can easily relapse. In our patient, we employed measures to prevent the recurrence of the condition. The preventive measures combined traditional Chinese and Western medicine, including RDATs, peanut red skin preparation, traditional Chinese medicine preparation, and ursodeoxycholic acid capsules and silybin capsules. Ursodeoxycholic acid capsules, silybin capsules and the traditional Chinese medicine preparation were added on the basis of these drugs. Ursodeoxycholic acid Capsules and silybin capsules were mainly used to protect the liver from the effects of long-term roxithromycin use. The patient's platelet count has been maintained at a normal level since March 2016, and recent laboratory examination results showed normal liver and kidney function.

The traditional Chinese medicine prescription given to the patient has the effects of relieving internal heat or fever and hemostasis, tonifying the liver and kidney, enriching essence and blood, strengthening the spleen and stomach, clearing away lung heat, and eliminating phlegm. Our patient was in an MCS and had been confined to bed for a long-time. She had decreasing lung function and was prone to sputum stagnation in the body, more phlegm, deficiency of Qi and Yin, and other symptoms. Therefore, Arisaema Cum Bile, Thunberg Fritillary Bulb, Bamboo Shavings, Bitter Apricot Kernel, and Rock White Mulberry were used to clean the lung and reduce phlegm. Herba Ecliptae, Glossy Privet Fruit, Barbary Wolfberry Fruit, Polygonatum Sibiricum, Glehniae Radix, Reed Rhizome, Dwarf Lilyturf Tuber, Radix Rehmanniae Preparata, and Reed Rhizome were used to moisten and tonify the lung and stomach, and to replenish essence and blood. The spleen and stomach are the basis of Qi and blood biochemistry. Qi and blood deficiency caused by long-term disease, only spleen and stomach functions recovery, Qi and blood biochemical having the source, could nourish internal organs, control blood. Therefore, Amomi Rotundus, Officinal Magnolia Bark, Common Aucklandiae Root, Immature Orange Fruit, Wrinkled giant hyssop herb, Chicken Cizzard Membrane, Medicinal Fermented Mass and so on were given to moisten Qi and warm the stomach, invigorate the spleen, and generate blood. Hemostasis was stopped with Garden Burnet Root and Cogongrass Rhizome. Patients in an MCS have deficiency of vital qi and low resistance, and often suffer from viral and other infections. Modern research has suggested that Honeysuckle flower, Forsythia Suspensa, Cyclamen, Heartleaf Houttuyniae Herba and Herba Oldenlandia can fight viruses and promote cellular immunity, and have an effect as a reinforcing agent (25).

In conclusion, MCS patients are prone to immune thrombocytopenia due to tracheotomy, long-term bed confinement, and decreased immunity. The present study showed that therapy combining traditional Chinese and Western medicine could cure immune thrombocytopenia and effectively prevent its recurrence. Western medicine 
to treat the infection could be selected according to the pathogen. Peanut red skin is a traditional Chinese medicine containing a variety of functional components that has the function of preventing thrombocytopenia, antibacterial and antioxidant effects. It has been recorded in National Compilation of Chinese Herbal Medicine for many years. This traditional Chinese medicine prescription has the effects of relieving internal heat or fever and hemostasis, tonifying the liver and kidney, enriching essence and blood, strengthening the spleen and stomach, clearing away lung heat, and eliminating phlegm. The traditional Chinese medicine given to the patient are both worth popularizing in clinic. Modern medicine should further strengthen the clinical development and utilization of Chinese traditional medicine, and the mechanism of action and molecular biology of traditional treatments should be studied further.

\section{Instructions}

Although our study was a case report, it could provide some valuable clues for the treatment of immune thrombocytopenia. Therefore, the next important step should be to carry out related causality experiments to further evidence the function of peanut red skin and explore the molecular biological mechanism.

\section{Acknowledgments}

Thanks to the medical and nursing team of the affiliated Hospital of Panzhihua University, and the relevant medical and technical departments.

Funding: None.

\section{Footnote}

Reporting Checklist: The authors have completed the CARE reporting checklist. Available at http://dx.doi.org/10.21037/ apm-21-307

Conflicts of Interest: All authors have completed the ICMJE uniform disclosure form (available at http://dx.doi. org/10.21037/apm-21-307). The authors have no conflicts of interest to declare.

Ethical Statement: The authors are accountable for all aspects of the work in ensuring that questions related to the accuracy or integrity of any part of the work are appropriately investigated and resolved. All procedures performed in studies involving human participants were in accordance with the ethical standards of the institutional and/or national research committee(s) and with the Helsinki Declaration (as revised in 2013). Written informed consent was obtained from the patient.

Open Access Statement: This is an Open Access article distributed in accordance with the Creative Commons Attribution-NonCommercial-NoDerivs 4.0 International License (CC BY-NC-ND 4.0), which permits the noncommercial replication and distribution of the article with the strict proviso that no changes or edits are made and the original work is properly cited (including links to both the formal publication through the relevant DOI and the license). See: https://creativecommons.org/licenses/by-nc-nd/4.0/.

\section{References}

1. Hodelín-Tablada R. Minimally conscious state: evolution of concept, diagnosis and treatment. MEDICC Rev 2016;18:43-6.

2. Claushuis TAM, de Vos AF, Nieswandt B, et al. Platelet glycoprotein VI aids in local immunity during pneumoniaderived sepsis caused by gram-negative bacteria. Blood 2018;131:864-76.

3. Chen M, Yan R, Zhou K, et al. Akt-mediated platelet apoptosis and its therapeutic implications in immune thrombocytopenia. Proc Natl Acad Sci U S A 2018;115:E10682-91.

4. Chen P, Chen SX, MA SQ. Correlation between Mycoplasma Pneumonia Infection and Newly Diagnosed of Immune Thrombocytopenia. Journal of Hubei University of Science and Technology (Medical Sciences) 2016;30:296-7.

5. Liu SX, Zhou LL, Zhou MQ. Determination and significance of mycoplasma pneumonia in children with immune thrombocytopenia. Chin J Health Lab Technol 2018;28:2505-6.

6. Sugiyama MG, Gamage A, Zyla R, et al. Influenza virus infection induces platelet-endothelial adhesion which contributes to lung injury. J Virol 2015;90:1812-23.

7. Yang J, Wang JM. Clinical observation on 34 cases of Thrombocytopenia in elderly patients with Pulmonary infection complicated with multiple Organ failure. Gansu Science and Technology 2017;33:97-8.

8. Stasi R. Pathophysiology and therapeutic options in primary immune thrombocytopenia. Blood Transfus 
2011;9:262-73.

9. Pishko AM, Lefler DS, Gimotty P, et al. The risk of major bleeding in patients with suspected heparin-induced thrombocytopenia. J Thromb Haemost 2019;17:1956-65.

10. Wang ML, Wang W. Current status of treatment for elderly patients with immune thrombocytopenia. J Clin Hematol (China) 2018;31:398-401.

11. Chen F, Zhu ZY. Liao GY. Progress in the treatment of Mycoplasma pneumoniae infection. Int J Lab Med 2017;38:2429-31.

12. Zheng X, Lee S, Selvarangan R, et al. MacarolideResistant Mycoplasma pneumonia. United State. Emerg Infect Dis 2015;21:1470-2.

13. Ling GT. Polyphenols, known as "the seventh kind of nutrient elements". China Food Addit. 2000;(1):28-37.

14. Wang J. Risk factors and countermeasures of the critically ill patients with Pulmonary infection caused by tracheotomy in intensive Care Unit. CNR 2014;(28):1873-74.

15. Yao JX. Virus infection associated thrombocytopenia and its pathogenesis. Foreign Medical Transfusion and Hematology Fascicles 2001;24:45-7.

16. Chen GX. Clinical observation on 38 cases of Thrombocytopenic Purpura treated with Peanut coating and Panax notoginseng. Yunnan Journal of traditional Chinese Medicine 2002;23:13-4.

17. Ren YH, Wu HB. Detection Method and Pharmacdogocal Functions of Reservratrol. J Anhui Agri Sci 2012;40:788-90.
18. Liu M. Study on the functional components of Peanut red enrich blood Oral liquid and its Toxicology Evaluation. Changchun University of Technology 2014.

19. Gao YT, Bao SZ, Zhang YJ. Study on peanut red skin in treating chemotherapy-induced thrombocytopenia by downregulating the expression of Bax, Bak protein. China J Tradit Chin Med Pharm 2018;33:3883-6.

20. Yan D, Zhao CB, Xie DS. Research Progress on Development and Utilization of Peanut Red clothing. Asia-Pacific Traditional Medicine 2015;11:51-4.

21. Zhang HW. Studies on the Chemical constituents, Derivatives and Biological activities of procyanidins in Peanut Red clothing. Inner Mongol University, 2014.

22. Dong W. Comparison of roxithromycin and azithromycin in the treatment of bacterial respiratory tract infection. Contemporary Medical Theories 2018;16:135-6.

23. Ma ZH, Sun CX, Hao DL. Immunomodulatory effect of ursodeoxycholic acid capsule in the treatment of cholestatic liver disease. Lab Med 2019;8:31-3.

24. Wang L. Ursodeoxycholic acid capsule combined with silibin capsule treated 30 cases of primary biliary cirrhosis. Chinese Medical Guide 2016;14:118-9.

25. Chen HX. Research Progress of traditional Chinese Medicine against infection. Gansu Science and Technology 2019;35:62-3.

(English Language Editor: J. Reynolds)

Cite this article as: Wang Y, Zhang L, Tian J, Lan M, Liu Y, Yan T, Qiu Q. The treatment of integrated traditional Chinese and Western medicine to cure severe immune thrombocytopenia in a patient in a minimally conscious state: a case report. Ann Palliat Med 2021;10(3):3483-3490. doi: 10.21037/apm-21-307 